\title{
KILLING KNOTS
}

P. M. RICE ${ }^{1}$

Let $k$ be a tame knot in $S^{3}, N(k)$ a regular neighborhood of $k$ and $M=S^{3} \backslash$ Int $N(k) . M$ may be collapsed [1] to a 2-complex $K$. Let $D$ be a disk in $N(k)$ with $D \cap \partial N(k)=\partial D$ and $\partial D$ not contractible on $\partial N(k) . M=N(K)$ is the mapping cylinder $C_{f}$ of a map $f: \partial N(K) \rightarrow K$, so $D \cup C_{f \mid \partial D} \cup K=L$ is a spine for $M \cup N(D)=U$. $\partial U$ is a 2-sphere, so $U$ is a cell and $L$ is cellular. Then $S^{3} / L \cong S^{3} . k \cap L$ is a single point in the interior of $D$ and $k \cap\left(S^{3} \backslash\right.$ Int $\left.U\right)$ is unknotted in $S^{3} \backslash$ Int $U$. It is easy to see that

THEOREM 1. If $k$ is a tame knot in $S^{3}$, there is a cellular 2-complex $L$ in $S^{3}$ such that $k \cap L$ is a single point, and under the projection $p: S^{3} \rightarrow S^{3} / L \cong S^{3}, p(k)$ is a tame and unknotted simple closed curve.

Note that if $k \cap L=\varnothing$, then $p(k)$ is unknotted if and only if $k$ is unknotted.

THEOREM 2. If $G=\pi_{1}\left(S^{3} \backslash k\right)$ is a knot group, there is a metric $d$ on $E^{3}$ (inducing the standard topology) and a closed set $P$ homeomorphic to $E^{1}$ such that $P$ is "straight" (for any three points $x, y, z \in P, d(x, y)$ $\pm d(y, z)=d(x, z))$ and $\pi_{1}\left(E^{3} \backslash P\right)=G$.

Proof. It may be assumed that $k \cap U=k \cap N(D)$ is a straight line segment. Let $P=k \cap$ Int $U$. Int. $U=E^{3}$ and inherits its metric from $S^{3}$. It is clear from the construction that $\pi_{1}$ (Int $\left.U \backslash P\right)=\pi_{1}\left(S^{3} \backslash k\right)$.

\section{REFERENCE}

1. J. H. C. Whitehead, Simplicial spaces, nuclei, and m-groups, Proc. London Math. Soc. 45 (1939), 243-327.

UNIVERSITY OF BONN AND UNIVERSITY OF GEORGIA

Received by the editors May 17, 1967.

1 The author is an Alexander von Humboldt Fellow and a Sarah Moss Fellow. 